\title{
ON A GENERALIZED VERSION OF THE NAKAYAMA CONJECTURE
}

\author{
MAURICE AUSLANDER ${ }^{1}$ AND IDUN REITEN
}

ABSTRACT. Nakayama proposed a conjecture which is equivalent to the following: If $\boldsymbol{\Lambda}$ is a finite dimensional algebra over a field and the dominant dimension of $\boldsymbol{\Lambda}$ is infinite, then $\boldsymbol{\Lambda}$ is self-injective. In this paper we study a generalized version of this conjecture.

Introduction. In [5] Nakayama proposed a conjecture, which by results of Müller [4] is equivalent to the following: If $\Lambda$ is a finite dimensional algebra over a field $k$, and $\operatorname{dom} \operatorname{dim} \Lambda=\infty$, i.e., in a minimal injective resolution

$$
0 \rightarrow \Lambda \rightarrow E_{0} \rightarrow E_{1} \rightarrow \cdots \rightarrow E_{i} \rightarrow \cdots
$$

for $\Lambda$, all the $E_{i}$ are projective, then $\Lambda$ is self-injective.

We recall that a finitely generated (left) $\Lambda$-module $M$ is said to be ultimately closed, if in a minimal projective resolution for $M$, there is a kernel $K_{n}$, each of whose summands is the summand of an earlier kernel [3]. Müller showed, generalizing results of Nakayama [5] and Tachikawa [6], that Nakayama's conjecture holds for an algebra which is an endomorphism ring of an ultimately closed generator-cogenerator for some artin algebra [4]. Further, Tachikawa [7] showed that the Nakayama conjecture is true for an algebra which is the endomorphism ring of a generator-cogenerator over a group algebra $k G$, where $k$ is a field and $G$ a finite $p$-group.

We propose here another conjecture, of which the Nakayama conjecture is a special case. We assume throughout that $\Lambda$ is an artin algebra, i.e., an artin ring which is a finitely generated module over its center, and that all the modules are left modules.

Conjecture. In a minimal injective resolution (1) for an artin algebra $\Lambda$, each indecomposable injective $\Lambda$-module occurs as the summand of some $E_{i}$.

If $\operatorname{dom} \operatorname{dim} \Lambda=\infty$, and each indecomposable injective $\Lambda$-module is a summand of some $E_{i}$ in the injective resolution for $\Lambda$, then all the injective $\Lambda$-modules would be projective, hence $\Lambda$ would be self-injective.

Our generalized Nakayama conjecture is further seen to be equivalent to the following.

Received by the editors July 9, 1974 .

AMS (MOS) subject classifications (1970). Primary 16A46, 16A52; Secondary $16 \mathrm{~A} 60,16 \mathrm{~A} 62$.

Key words and phrases. Dominant dimension, self-injective ring, injective resolution.

1 This paper was written while the first author was partially supported by NSF GP-33406X1. 
If $S$ is a simple $\Lambda$-module with $\operatorname{Ext}_{\Lambda}^{i}(S, \Lambda)=0$ for $i \geq 0$, then $S$ is zero.

To see this, we use the fact that $\operatorname{Ext}_{\boldsymbol{\Lambda}}^{i}(S, \Lambda) \neq(0)$ if and only if $S \subset E_{i}$ (i.e., $E(S)$ is a summand of $E_{i}$ where $E(S)$ is the injective envelope of $S$ ).

For the sake of completeness, we mention that the Nakayama conjecture would be a consequence of the conjecture that the finitistic global dimension of any finite dimensional algebra over a field is finite. We recall that the finitistic global dimension is the supremum of $\mathrm{pd} M$, where $M$ runs through all modules with pd $M<\infty$. For if $\operatorname{dom} \operatorname{dim} \Lambda=\infty$, (1) is a minimal injective resolution for $\Lambda$, then all the kernels in this sequence have finite projective dimension. If there was an upper bound on these dimensions, $0 \rightarrow \Lambda \rightarrow E_{0}$ would split, so that $\Lambda$ would be self-injective.

In this paper we shall discuss the generalized Nakayama conjecture. We shall first show that this conjecture is true if and only if the following conjecture is true.

Conjecture. If $\Lambda$ is an artin algebra, and $M$ is a generator such that $\operatorname{Ext}_{\mathbf{\Lambda}}^{i}(M, M)=0$ for $i \geq 1$, then $M$ is projective.

The Nakayama conjecture is known to hold for every artin algebra if and only if for any artin algebra $\Lambda$, $M$ being a generator-cogenerator with $\operatorname{Ext}_{\boldsymbol{\Lambda}}^{i}(M, M)=0$ for $i \geq 1$, implies that $M$ is projective [4]. We obtain this result as a special case of our considerations.

We shall then show that in the various cases where we know that the Nakayama conjecture holds, the generalized Nakayama conjecture holds. This means that we get the same conclusion by weakening $M$ being a generator-cogenerator to $M$ being a generator.

Mod $\Lambda$ will denote the category of (left) $\Lambda$-modules, $\bmod \Lambda$ the category of finitely generated (left) $\Lambda$-modules.

1. Before we can reformulate our generalized Nakayama conjecture, we shall need to recall some useful results on rings of endomorphisms from [1] and [2].

We consider pairs $(\Lambda, M)$, where $\Lambda$ is a ring (i.e., here an artin algebra) and $M$ a finitely generated (left) $\Lambda$-module. We denote by End the map on such pairs given by $\operatorname{End}(\Lambda, M)=(\Gamma, P)$, where $\Gamma=\operatorname{End}_{\Lambda}(M)^{\mathrm{op}}$, and $P=$ $(M, \Lambda)$. A projective $\Gamma$-module $P$ is said to be a Wedderburn projective $\Gamma$ module if $\operatorname{End}^{2}(\Gamma, P)^{r}=\operatorname{End}(\operatorname{End}(\Gamma, P))=(\Gamma, P)$, and if $M$ is a (finitely generated) $\Lambda$-generator, then $P$ is a Wedderburn projective $\Gamma$-module and $\operatorname{End}^{2}(\Lambda, M)=(\Lambda, M)$. Hence End gives a one-one correspondence between pairs $(\Lambda, M)$, with $M$ a finitely generated $\Lambda$-generator, and $(\Gamma, P)$, with $P$ a a Wedderburn projective. If

$$
0 \rightarrow \mathrm{\Gamma} \rightarrow E_{0} \rightarrow E_{1} \rightarrow \cdots \rightarrow E_{i} \rightarrow \cdots
$$

is a minimal injective resolution for $\Gamma$, then $P$ is a Wedderburn projective if and only if for each simple submodule $S$ of $E_{0}$ II $E_{1}, P$ contains the projec- 
tive cover $P_{S}$ of $S$ as a summand. $P$ is said to be a minimal Wedderburn projective if the only indecomposable summands of $P$ are the above $P_{S}$. End gives a one-one correspondence between pairs $(\Lambda, M)$ and $(\Gamma, P)$, where $M$ is a (finitely generated) generator such that each indecomposable injective $\Lambda$-module occurs as a summand of $E_{0}(M) \amalg E_{1}(M)$, where $E_{0}(M)$ and $E_{1}(M)$ are the first two terms in a minimal injective resolution for $M$, and $P$ is a minimal Wedderburn projective $\Gamma$-module.

For $M$ a $\Lambda$-generator and $P$ a Wedderburn $\Gamma$-projective, the functors $\operatorname{Hom}_{\Gamma}(P):, \operatorname{Mod} \Gamma \rightarrow \operatorname{Mod} \Lambda$ and $\alpha=\operatorname{Hom}_{\Lambda}(M):, \operatorname{Mod} \Lambda \rightarrow \operatorname{Mod} \Gamma$ have the property that $\operatorname{Hom}_{\Gamma}(P,) \operatorname{Hom}_{\Lambda}(M):, \operatorname{Mod} \Lambda \rightarrow \operatorname{Mod} \Lambda$ is isomorphic to the identity functor. And if $a$ denotes the full subcategory of Mod $\Gamma$ whose objects are thus isomorphic to the $\Gamma$-modules $(M, X)$ for $X$ in $\operatorname{Mod} \Lambda$, then the restriction of $\operatorname{Hom}_{\Gamma}\left(P_{,}\right)$to $\alpha$ gives an equivalence of categories between $\alpha$ and Mod $\Lambda$.

There is another useful description of $a$. Let $E=E(P / x P)$ be the injective envelope of $P / x P$, wher e $x$ denotes the radical of $\Gamma$. Then the objects of $\alpha$ are the $\Gamma$-modules $Y$ such that $E_{0}(Y) \amalg E_{1}(Y)$ is in $\mathbf{V}(E)$, the full additive subcategory of Mod $\Gamma$ generated by $E$. An object $(M, X)$ in $\alpha$ is an injective $\Gamma$-module if and only if $X$ is an injective $\Lambda$-module.

We have

Theorem 1.1. (a) If the generalized Nakayama conjecture does not hold for $\Gamma$, choose $P$ to be a projective $\Gamma$-module whose indecomposable summands are exactly the $P_{S}$, where $S$ is a simple submodule of some $E_{i}(\Gamma)$. Then for $(\Lambda, M)=\operatorname{End}(\Gamma, P), M$ is a nonprojective generator such that $\operatorname{Ext}_{\Lambda}^{i}(M, M)$ $=0$ for $i \geq 1$.

(b) If $(\Lambda, M)$ is such that $M$ is a nomprojective generator and $\operatorname{Ext}_{\Lambda}^{i}(M, M)=$ 0 for $i \geq 1$, then for $(\Gamma, P)=$ End $(\Lambda, M)$, the generalized Nakayama conjecture does not hold for $\Gamma$.

Proof. (a) Assume that the generalized Nakayama conjecture does not hold for $\Gamma$, and let (2) be a minimal injective resolution for $\Gamma$. Let $P$ be as in the statement of the Theorem. The projective $\Lambda$-modules are the $(P, Q)$, where $Q$ is a summand of a finite sum of copies of $P$. Hence, by our assumption on $\Gamma, M=(P, \Gamma)$ is not a projective $\Lambda$-module. We now want to show that $\operatorname{Ext}_{\Lambda}^{i}(M, M)=(0)$ for all $i \geq 1$.

We have the exact sequence of $\Lambda$-modules

$$
0 \rightarrow M=(P, \Gamma) \rightarrow\left(P, E_{0}\right) \rightarrow\left(P, E_{1}\right) \rightarrow \cdots \rightarrow\left(P, E_{i}\right) \rightarrow \cdots
$$

Since all the $E_{i}$ clearly are in $a$, all the $\left(P, E_{i}\right)$ are injective $\Lambda$-modules. Consider now the sequence

$0 \rightarrow((P, \Gamma),(P, \Gamma)) \rightarrow\left((P, \Gamma),\left(P, E_{0}\right)\right) \rightarrow \cdots \rightarrow\left((P, \Gamma),\left(P, E_{i}\right)\right) \rightarrow \cdots$ 
Since $\Gamma$ and all the $E_{i}$ lie in $\alpha$, we use the equivalence of categories that $\operatorname{Hom}_{\boldsymbol{\Lambda}}(P)$ induces between $\alpha$ and $\operatorname{Mod} \Lambda$, to get that this sequence is isomorphic to the sequence (2) which we know is exact. It follows that $\operatorname{Ext}_{\boldsymbol{\Lambda}}^{i}(M, M)=0$ for $i \geq 1$.

(b) Let $(\Lambda, M)$ be such that $M$ is a nonprojective generator with $\operatorname{Ext}_{\Lambda}^{i}(M, M)=0$ for $i \geq 1$, and let $(\Gamma, P)=\operatorname{End}(\Lambda, M)$. Let

$$
0 \rightarrow M \rightarrow I_{0} \rightarrow I_{1} \rightarrow \cdots \rightarrow I_{i} \rightarrow \cdots
$$

be a minimal injective resolution for $M$. Hence we get that

$$
0 \rightarrow(M, M) \rightarrow\left(M, E_{0}\right) \rightarrow\left(M, E_{1}\right) \rightarrow \cdots \rightarrow\left(M, E_{i}\right) \rightarrow \cdots
$$

is an injective resolution for $\Gamma$. The number of nonisomorphic indecomposable injective $\Gamma$-modules is strictly larger than the corresponding number for $\Lambda$, since $M$ is a nonprojective generator. But the number of indecomposable injective $\Gamma$-modules which are summands in a minimal injective resolution for $\Gamma$ can be at most this smaller number.

Corollary 1.2. In the Wedderburn correspondence between $(\Lambda, M)$ and $(\Gamma, P), M$ is a generator-cogenerator with $\operatorname{Ext}_{\Lambda}^{i}(M, M)=0$ for $i \geq 1$ if and only if $\operatorname{dom} \operatorname{dim} \Gamma=\infty$ (and $P$ is a minimal Wedderburn projective). And under this correspondence, $M$ is projective if and only if $\Gamma$ is self-injective.

Proof. If $\operatorname{dom} \operatorname{dim} \Gamma=\infty$, the previous choice of $P$ is a minimal Wedderburn projective, since in this case all the indecomposable summands of the $E_{i}(\Gamma)$ are already summands of $E_{0}(\Gamma) \amalg E_{1}(\Gamma)$. Since all the injective $\Gamma$-modules which lie in $a$ are projective $\Gamma$-modules, $M=(P, \Gamma)$ contains all indecomposable injective $\Lambda$-modules as summands, i.e., $M$ is a cogenerator.

And if conversely $M$ is a generator-cogenerator with $\operatorname{Ext}_{\boldsymbol{\Lambda}}^{i}(M, M)=0$ for $i \geq 1$, then for each indecomposable injective $\Lambda$-module $I,(M, I)$ is a projective $\Gamma$-module, so that $\operatorname{dom} \operatorname{dim} \Gamma=\infty$.

Since $M$ is a $\Lambda$-generator-cogenerator, we have for the pair $(\Gamma, P)=$ End $(\Lambda, M), P$ is a minimal Wedderburn projective $\Gamma$-module, so that we can use this $P$ for the opposite direction.

If $M$ is projective, then $\Lambda$, hence $\Gamma$, is self-injective. And we have seen that if $M$ is not projective, the generalized Nakayama conjecture does not hold for $\Gamma$, so that $\Gamma$ is not self-injective.

While the Nakayama conjecture is consequently true if and only if $M$ being a $\Lambda$-generator-cogenerator with $\operatorname{Ext}_{\Lambda}^{i}(M, M)=0$ for $i \geq 1$ implies that $M$ is projective, the generalized Nakayama conjecture is true if and only if the following conjecture is true.

Conjecture. If $\Lambda$ is an artin algebra and $M$ a $\Lambda$-generator such that $\operatorname{Ext}_{\Lambda}^{i}(M, M)=0$ for $i \geq 1$, then $M$ is projective.

We shall see that, in this form, the generalized Nakayama conjecture is 
known in the same situations as the ordinary one. If $\Lambda$ is self-injective, they are the same. Here Tachikawa [7] has given an affirmative answer for a group algebra $k G$ for $k$ a field, when $G$ is a finite $p$-group. The other cases are given by Müller [4], and the following result is a generalization to our situation.

Proposition 1.3. If $M$ is a (finitely generated) $\Lambda$-generator which is ultimately closed and such that $\operatorname{Ext}_{\boldsymbol{\Lambda}}^{i}(M, M)=0$ for $i>1$, then $M$ is projective.

Proof. We shall find useful the general

Lemma 1.4. If $M$ is a finitely generated generator for an artin algebra $\Lambda$, with $\operatorname{Ext}^{i}(M, M)=0$ for $i \geq 1$ then for each syzygy module $N=\Omega^{n} M$ of $M, \operatorname{Ext}_{\mathbf{\Lambda}}^{i}(N, N)=0$.

Proof of the Lemma. Since $M$ is a generator, $\operatorname{Ext}_{\Lambda}^{i}(M, \Lambda)=0$. From this we can conclude that $\operatorname{Ext}^{i+n}(M, N) \simeq \operatorname{Ext}^{i}(M, M)$ for $i \geq 1$. We also have $\operatorname{Ext}^{i}(N, N) \simeq \operatorname{Ext}^{i+n}(M, N)$ for $i \geq 1$. Hence we conclude that $\operatorname{Ext}^{i}(N, N)=0$ for $i \geq 1$, since $\operatorname{Ext}^{i}(M, M)=0$ for $i \geq 1$. This finishes the proof of the Lemma.

Returning to the proof of the proposition, we know that since $M$ is ultimately closed, there is some $N=\Omega^{n} M$, all of whose summands occur as a summand in some earlier $\Omega^{j} M$ (i.e., $j<n$ ). If $M$ is not projective, then pd $M=\infty$, since $\operatorname{Ext}^{i}(M, \Lambda)=0$ for $i \geq 1$. Then we would have pd $N=\infty$, hence pd $N^{\prime}=\infty$ for some indecomposable summand $N^{\prime}$ of $N$. But then some summand $N^{\prime \prime}$ of $N$ is a kernel in a minimal projective resolution for $N^{\prime}$, so that $\operatorname{Ext}^{j}\left(N^{\prime}, N^{\prime \prime}\right) \neq 0$ for some $j \geq 1$. Hence $\operatorname{Ext}^{j}(N, N) \neq 0$ for some $j \geq 1$, which is a contradiction. This finishes the proof of the proposition.

Since the ultimately closed algebras play a central role in these investigations, we shall mention some examples of such algebras: If for some $k \geq 0, \Lambda$ has a finite number of indecomposable modules which are summands of $k$ th syzygy modules, then $\Lambda$ is ultimately closed.

As special cases of this class we have: $\Lambda$ of finite representation type, gl $\operatorname{dim} \Lambda<\infty$, the square of the radical of $\Lambda$ is zero, etc.

We end this note with the following observation concerning the generalized Nakayama conjecture.

Proposition 1.5. The following statements are equivalent;

(a) The generalized Nakayama conjecture is true for all artin algebras.

(b) If $\Lambda$ is an artin algebra with the property that for each simple $\Lambda$. module $S$ there is an integer $i$ such that $\operatorname{Ext}^{i}(S, \Lambda) \neq 0$, then a finitely generated $\Lambda$-generator $M$ is projective provided $\operatorname{Ext}_{\Lambda}^{i}(M, M)=0$ for all $i \geq 1$.

Proof. (a) implies (b). Already shown. 
(b) implies (a). For each artin algebra $\Gamma$ let $m(\Gamma)$ be the number of nonisomorphic simple $\Gamma$-modules. Let $\mathcal{T}$ be the collection of artin algebras with the property that $\Gamma$ is in $\mathcal{T}$ if and only if $n(\Gamma)<m(\Gamma)$ where $n(\Gamma)$ is the number of nonisomrophic simple $\Gamma$-modules $S$ such that $\operatorname{Ext}_{\Gamma}^{i}(S, \Gamma) \neq 0$ for some $i \geq 0$. We want to show that $\mathcal{T}=\varnothing$ in order to show that (b) implies (a).

Suppose $\mathcal{T} \neq \varnothing$. Let $\Gamma$ be an artin algebra in $\mathcal{T}$ with $m(\Gamma)$ as small as possible. Let $S_{1}, \ldots, S_{n(\Gamma)}$ be a set of nonisomorphic simple $\Gamma$-modules such that for each $S_{j}$ there is an integer $i_{j}$ with the property $\operatorname{Ext}_{\Gamma}{ }_{\Gamma}\left(S_{j}, \Gamma\right) \neq$ 0 . Let $P_{j}$ be a projective cover for $S_{j}$ and let $P=P_{1} \amalg \cdots \amalg P_{n(\boldsymbol{r})}$ (direct sum). Finally, let End $(\Gamma, P)=(\Lambda, M)$.

Clearly $m(\Lambda)=n(\Gamma)<m(\Gamma)$. Hence $\Lambda$ is not in $\mathcal{J}$ so that $\Lambda$ has the property that for each simple $\Lambda$-module $S$ we have that $\operatorname{Ext}_{\Lambda}^{i}(S, \Lambda) \neq 0$ for some $i \geq 0$. Hence by (b), a $\Lambda$-generator $N$ is projective if $\operatorname{Ext}^{i}(N, N)=0$ for all $i \geq 1$. But by Theorem $1.1, M$ is a $\Lambda$-generator with the property $\operatorname{Ext}^{i}(M, M)=0$ for all $i \geq 1$ which is not $\Lambda$-projective. This contradiction shows that $\mathcal{T}=$ which shows that (b) implies (a).

In other words, to prove that all artin algebras $\Lambda$ have the property that a $\Lambda$-generator $M$ is projective if $\operatorname{Ext}_{\Lambda}^{i}(M, M)=0$ for all $i \geq 1$, it suffices to prove it only for artin algebras $\Lambda$ with the property that given a simple $\Lambda$-module $S$, there is an integer $i$ such that $\operatorname{Ext}^{i}(S, \Lambda) \neq 0$.

\section{REFERENCES}

1. M. Auslander, Representation theory of artin algebras. I, Comm. Algebra 1 (1974), 177-268.

2. M. Auslander and I. Reiten, Stable equivalence of dualizing R-varieties, V: Artin algebras stably equivalent to hereditary algebras, Advances in Math. (to appear).

3. J. P. Jans, Some generalizations of finite projective dimension, Illinois J. Math. 5 (1961), 334-344. MR 32 \#1226.

4. B. J. Mueller, The classification of algebras by dominant dimension, Canad. J. Math. 20 (1968), 398-409. MR 37 \#255.

5. T. Nakayama, On algebras with complete homology, Abh. Math. Sem. Univ. Hamburg 22 (1958), 300-307. MR 21 \#3471.

6. H. Tachikawa, On dominant dimensions of QF-3 algebras, Trans. Amer. Math. Soc. 112 (1964), 249-266. MR 28 \#5092.

7. - Quasi-Frobenius rings and generalizations, Lecture Notes in Math., vol. 351, Springer-Verlag, Berlin and New York, 1973.

DEPARTMENT OF MATHEMATICS, BRANDEIS UNIVERSITY, WALTHAM, MASSACHUSETTS 02154

DEPARTMENT OF MATHEMATICS, MASSACHUSETTS INSTITUTE OF TECHNOLOGY, CAMBRIDGE, MASSACHUSETTS 02139

DEPARTMENT OF MATHEMATICS, UNIVERSITY OF TRONDHEIM, TRONDHEIM, NORWAY 